\title{
P04-23. HIV-I neutralization is impacted by the PBMC donor used for both virus growth and target cells, and the effects are neutralization reagent-specific
}

\author{
L Wieczorek*1, BK Brown ${ }^{1}$, M Wesberry ${ }^{1}$, C Ochsenbauer-Jambor ${ }^{2}$, \\ JC Kappes ${ }^{2}$, N Lora ${ }^{1}$, A Gillis ${ }^{1}$, C Nzodom ${ }^{1}$, S Molnar ${ }^{1}$, NL Michael ${ }^{3}$, \\ D Montefiori ${ }^{4}$ and VR Polonis ${ }^{3}$
}

Address: ${ }^{1}$ Retrovirology, US Military HIV Research Program/Henry M. Jackson Foundation, Rockville, MD, USA, ${ }^{2}$ University of Alabama, Birmingham, AL, USA, ${ }^{3}$ US Military HIV Research Prgm/Walter Reed Army Institute of Research, Rockville, MD, USA and ${ }^{4}$ Duke University, Durham, NC, USA

* Corresponding author

from AIDS Vaccine 2009

Paris, France. 19-22 October 2009

Published: 22 October 2009

Retrovirology 2009, 6(Suppl 3):P5 I doi: I0.II86/1742-4690-6-S3-P5 I

This abstract is available from: http://www.retrovirology.com/content/6/S3/P5 I

(C) 2009 Wieczorek et al; licensee BioMed Central Ltd.

\section{Background}

The assessment of HIV neutralization using peripheral blood mononuclear cells (PBMC) is a complex process, attributable to numerous variables. Here we measure both intra- and inter-assay variability in a PBMC neutralization assay, and assess the variation contributed by the PBMC source for both virus propagation and assay targets, in efforts to inform modifications required for assay standardization.

\section{Methods}

Primary isolates or infectious molecular clones (IMC) were propagated in PBMC from multiple donors (or were 293T cell-derived); neutralization profiles were assessed using different target cells. Cell line targets were used to further assess the influence of virus source, and reporter IMCs tested assay design and endpoint variation. The endpoints were p24 production or Renilla luciferase expression; $\mathrm{IC}_{50}$ ranges were compared to determine intra- and inter-assay variability.

\section{Results}

Intra-assay variation (between operators using the same reagents) was independent of reagents used and averaged 2.2-fold ( $\mathrm{N}=253$ assays, excluding concordant negative results). Altering the source of PBMC targets induced inter-assay variability that was neutralization reagent-specific, and occurred even when 293T-derived virus was used. The greatest differences were observed with $2 \mathrm{G} 12$ (194-fold range in $\mathrm{IC}_{50 \mathrm{~s}}$ ) and ten-fold less variation was observed using sCD4 (11-fold maximum range). The variability resulting from using different targets was not reduced by employing novel, luciferase-expressing IMC. Varying the PBMC source for viral propagation also induced inter-assay variation, and viral strains behaved differently. Using reporter cell line targets with virus produced in different PBMC limited inter-assay differences to that of intra-assay variation.

\section{Conclusion}

Variability in the PBMC neutralization assay is influenced by the PBMC source and multiple variables. While use of reporter cell targets reduced neutralization assay variability, novel reporter IMCs reduce PBMC assay workload but not the variability related to donor differences. Further analysis of host genetic properties will facilitate identification of specific characteristics that significantly influence neutralization profiles. 